\title{
A PERCEPÇÃO DOS PROFISSIONAIS DE ENFERMAGEM SOBRE O USO DA LISTA DE VERIFICAÇÃO DE SEGURANÇA CIRÚRGICA
}

\author{
Perception of nursing professionals on the use of surgical safety checklist
}

La percepción del equipo de enfermería sobre la utilización de la lista de verificación de seguridad cirúrgica

Priscila Thais Leme Alves Ferreira ${ }^{1}$, Aline Eloá Barbosa ${ }^{2}$, Nathalia Malaman Galhardi ${ }^{3}$, Thalyta C. A. Teixeira ${ }^{4}$

\section{Resumo}

O estudo teve por objetivo avaliar o conhecimento de equipe de enfermagem sobre a aplicabilidade e importância do Programa Cirurgia Segura e da utilização da Lista de Verificação de Segurança Cirúrgica. Trata-se de estudo descritivo e quantitativo realizado no centro cirúrgico de um hospital universitário público envolvendo uma população de 34 profissionais, cujos dados foram coletados por meio de questionário estruturado. Participaram da pesquisa 26 auxiliares de enfermagem e dois enfermeiros. O estudo destacou aspectos positivos quanto ao conhecimento e à credibilidade dada pelos profissionais em relação ao uso da Lista de Verificação em questão como ferramenta. Os profissionais também apresentaram as dificuldades decorrentes do uso dessa

\footnotetext{
${ }^{1}$ Enfermeira, Hospital Estadual de Sumaré, endereço Rua Miguel Guidotti,405, Egistro Ragazzo, Limeira/SP, Email:prialfer12@hotmail.com, Telefone: 19981105923.

2 Especialista em terapia Intensiva, Enfermeira, Universidade Paulista - UNIP Campus Limeira, endereço Rua Papa João Paulo II,358, Orlando Correa, Artur Nogueira/SP, E- mail: lieloa_b@hotmail.com, Telefone: 19 99153-5004.

${ }^{3}$ Mestre em Ciências da Saúde, Enfermeira, Universidade Paulista UNIP Campus Limeira, Enfermeira Especialista em Urgência e Trauma, endereço Rua Santa Cruz, 1150, Centro Limeira/SP. Email: nathymalaman@hotmail.com, Telefone: 19982555734.

4 Doutora em Ciências da Saúde, Enfermeira, Universidade Paulista UNIP , endereço Rua Monsenhor Fergo O'Connor de Camargo Dauntre, 401- Vila Joaquim Inacio, Campinas/ SP. Email: thalytacat@yahoo.com.br, Telefone: 19 99333-0147.

Rev. Gestão \& Saúde (Brasília) Vol. 10, n. 01, Jan. 2019.
}

Lista tais como a ausência de comunicação verbal entre a equipe em relação ao paciente e de tempo para a conferência dos instrumentos antes da cirurgia. Os resultados permitiram destacar, analisar e discutir sobre as lacunas que ainda permeiam o processo de adesão da Lista de Verificação na rotina do centro cirúrgico. São necessários o planejamento e a implantação de atitudes que contribuam para que a Lista de Verificação seja adotada como ferramenta para a segurança do paciente cirúrgico.

Descritores: Centros Cirúrgicos; Gerenciamento de Segurança; Lista de Checagem, Enfermagem.

\begin{abstract}
The purpose of this study was to evaluate nursing team knowledge about the applicability and importance of the Safe Surgical Program and the use of the Surgical Safety Checklist. It is a descriptive and quantitative study carried out in the surgical center of a public university hospital involving a population of 34 professionals, whose data were collected by means of a structured questionnaire. Twenty-six nursing assistants and two nurses participated in the study. The study highlighted positive aspects as for the knowledge and credibility granted by professionals as to the Surgical Safety Checklist use as a tool. The professionals also referred to the
\end{abstract}


Revista Eletrônica Gestão \& Saúde ISSN: 1982-4785

Ferreira PTLA, TeixeiraTCA, Galhardi, NM et al

difficulties arising from the use of such Checklist, as the absence of verbal communication among the team concerning the patient and available time for the checking of the instruments before the surgery. The results allowed to highlighting, analyzing and discussing the gaps still permeating the process of adherence to the Checklist in the surgical center routine. It is necessary to plan and implement systems that contribute to the Checklist adoption as a tool for the safety of the surgical patient.

Descriptors: Surgicenters; Safety Management; Cehcklist; Nursing.

\section{Resumen}

Este trabajo tuvo como objetivo evaluar el conocimiento del equipo de enfermería sobre la aplicación e importancia del Programa de la Cirurgia Segura y la utilización de la lista de verificación de seguridad cirúrgica. Se realizo un estudio descritivo, cualitativo en el centro cirúrgico de un hospital universitario público, durante el período de junio a julio del 2015, el universo estuvo constituído por 34 profesionales,

\section{Introdução}

A qualidade da assistência à saúde é determinada por um processo complexo que inclui o julgamento dos profissionais e usuários em que os avanços no setor saúde ocorrem articuladamente às possibilidades de transformação geral da sociedade, aos custos da assistência, bem como à necessidade de promover a equidade de acesso, os esforços das instituições direcionam-se no sentido de encontrar evidências do seu desempenho para atigir a qualidade dos seus serviços1.

Rev. Gestão \& Saúde (Brasília) Vol. 10, n. 01, Jan. 2019. participaron en la encuesta 26 auxiliares de la enfermeria y dos enfermeros, los datos fueron recolectados mediante un cuestionario estructurado. El estudio destacó los aspectos positivos en cuanto al conocimiento y la credibilidad dada por los profesionales de la salud en relación a la utilización de la lista de verificación como una herramienta fundamental. Los profesionales presentaron dificultadesenel usodelalista, como la falta de comunicación verbal entre el equipo y el paciente y falta de tiempo para la conferencia de los instrumentos antes de la cirurgía. La conclusión de este trabajo basándonos en los resultados, fue posible destacar, analizar y discutir sobre las fallas que todavia existen en el proceso de adesión de la lista de verificación de Seguridad Cirúrgica en la rutina del Centro Quirúrgico. Se hace necesario la planificación y implementación de estratégias que ayuden para que la lista de verificación sea adoptada como una importante herramienta para la seguridad del paciente quirúrgico.

Descriptores: Centros Quirúrgicos; Gerenciamento de Seguridad; Lista de Verificación, Enfermería.

A atenção da assistência à saúde, voltada para a segurança do paciente e à prevenção de eventos adversos (EAs),

tornou-se um movimento mundial, para o qual as instituições de saúde que defendem a qualidade vêm direcionando seu foco.

Define-se segurança do paciente como a prevenção de erros e efeitos adversos aos pacientes associados aos cuidados de saúde. Trata-se de um componente crítico da qualidade dos cuidados de saúde e é muitas vezes descrita como pré-requisito para um atendimento de alta qualidade2. 
Revista Eletrônica Gestão \& Saúde ISSN: 1982-4785 Ferreira PTLA, TeixeiraTCA, Galhardi, NM et al

Quando se fala em ações voltadas para a promoção da segurança do paciente, é necessário destacar intervenções que contribuem para o sucesso na prestação de um cuidado seguro, como, por exemplo, a prevenção dos EAs, definidos como complicações indesejadas decorrentes do cuidado prestado aos pacientes, não atribuídas à evolução natural da doença de base e que podem causar danos3.

Quanto à atuação da equipe de enfermagem no perioperatório, entendese que esses profissionais exercem papel complexo, pois, além da necessidade de conhecimento científico sobre o atendimento ao paciente, necessitam saber como articular atividades relacionadas ao trabalho em equipe, o que envolve relacionamento interpessoal, geralmente dificultado em unidades de trabalho fechadas, onde várias profissões da saúde interagem4.

Revisão da Literatura

As especificidades do período perioperatório foram muito discutidas ao longo do tempo. Sobre as ações a ele direcionadas, destaca-se o lançamento, na 57a Assembléia Mundial da Saúde, em 2004, da Aliança Mundial para a Segurança do Paciente com o intuito de construir politicas e práticas clínicas seguras5.

Implementaram-se ações para a redução dos EAs advindos de práticas não seguras de cuidados. Para esses problemas, foram lançadas campanhas de segurança do paciente chamadas 'Desafios Globais para a Segurança do Paciente'. O Primeiro Desafio Global trabalhado nos anos de 2005 e 2006 foi sobre 'Infecção associada aos cuidados de saúde', no qual foram formados padrões simples e claros para a higiene das mãos, campanha educativa e as primeiras orientações da Organização Mundial de Saúde (OMS) acerca do assunto. Já o Segundo Desafio Global, entre 2007 e 2008, foi a segurança dos cuidados cirúrgicos, sendo elaboradas diretrizes sobre cirurgia segura5.

A OMS elaborou o programa 'Cirurgia Segura Salva Vidas', criando, para isso, a Lista de Verificação de Segurança Cirúrgica (Lista de Verificação), oficialmente lançada em Washington DC, Estado Unidos, em 25 de junho de 2008, com o objetivo de promover sua adoção e uso mundial6.

No caso do Brasil, material divulgado pela Agência Nacional de Vigilância Sanitária (Anvisa)7 destaca que houve uma iniciativa conjunta da Secretaria de Atenção à Saúde, a própria Anvisa e a Organização Pan-americana da Saúde (Opas). Todas reuniram esforços para a viabilização do projeto 'Segurança do Paciente e Qualidade em Serviços de Saúde - Cirurgias Seguras Salvam Vidas'.

Em 2013, a ANVISA lançou o Programa Nacional de Segurança do Paciente (PNSP). Seu objetivo é o de promover e apoiar a implantação de iniciativas voltadas à segurança do paciente em diferentes áreas da atenção, organização e gestão de serviços de saúde por meio da implantação da gestão de risco e de Núcleos de Segurança do Paciente nos estabelecimentos de saúde do país8.

Dentre as ações e a elaboração de técnicas voltadas à prevenção de EAs para os pacientes submetidos à cirurgia, apresentadas na campanha 'Cirurgias Seguras Salvam Vidas', destaca-se a Lista de Verificação. Seu objetivo é o de assegurar que elementos-chave de segurança sejam incorporados à rotina da sala de operações. Tal decisão maximizará a chance de melhores resultados para os pacientes sem que ocorra ônus indevido ao sistema e aos 
Revista Eletrônica Gestão \& Saúde ISSN: 1982-4785 Ferreira PTLA, TeixeiraTCA, Galhardi, NM et al

prestadores9. É uma ferramenta para ser utilizada por profissionais com interesse na melhoria da segurança cirúrgica e na redução de complicações evitáveis6.

Estudo que avaliou a efetividade da lista de verificação de segurança cirúrgica em cirurgias eletivas realizadas em oito países revelcou que as grandes complicações foram reduzidas de $11 \%$ para $7 \%(\mathrm{p}<0,001)$, a mortalidade caiu de $1,5 \%$ para $0,8 \%(p=0,003)$ e a adesão à antibioticoprofilaxia aumentou de 55\% para $83 \% 9$.

Em relação à aplicabilidade da Lista de Verificação e do protocolo para a cirurgia segura, a equipe de enfermagem precisa saber exatamente por que está aplicando, e assim, ter argumentos para provar e defender a importância dessas ferramentas.

Pretendeu-se com este estudo contribuir para o fortalecimento da linha de pesquisa que trata da segurança do paciente e da prevenção de eventos adversos, contemplando recursos humanos, qualidade e organização dos serviços.

Teve-se como objetivo avaliar o conhecimento da equipe de enfermagem sobre a aplicabilidade e importância da adoção do Programa Cirurgia Segura (PCS), assim como a utilização da Lista de Verificação no centro cirúrgico de hospital universitário.

Método

Trata-se depesquisa transversal e descritiva com abordagem quantitativa, realizada no centro cirúrgico de hospital universitário de grande porte localizado no interior do Estado de São Paulo, acreditado pela Organização Nacional de Acreditação (ONA), nível III, e pela Acreditação Internacional Canadense 10 . A população do estudo envolveu 34 profissionais atuantes na equipe de enfermagem do centro cirúrgico, sendo que a amostra foi composta por 28 deles, dois dos quais ocupavam o cargo de enfermeiro e 26 eram auxiliares de enfermagem.

A coleta de dados foi realizada no período de 20 de junho a 10 de julho de 2015 por meio de questionário autoaplicável previamente construído e validado em outra pesquisa 10 .

A fim de obter o maior número de respostas, não se realizou o cálculo amostral, ou seja, o questionário foi aplicado a todos que concordaram com os critérios de inclusão e exclusão preestabelecidos.

Incluíram-se enfermeiros e auxiliares de enfermagem que atuavam no centro cirúrgico durante o período da coleta de dados e excluíram-se aqueles que estavam de férias ou licença no período.

Os dados foram compilados em uma tabela utilizando o programa Microsoft Excel (2011) e as divergências na alimentação dos dados foram verificadas manualmente e corrigidas. Excluíram-se os questionários com mais de $20 \%$ das questões não respondidas.

Os resultados da pesquisa foram apresentados sob a forma descritiva, ilustrados por gráficos e tabelas.

$\mathrm{O}$ projeto de pesquisa foi realizado de acordo com as Diretrizes e Normas da Resolução no 466/12 de 12 de Dezembro de 2012, estabelecidas pelo Conselho Nacional de Saúde, defendendo os interesses dos sujeitos em sua integridade e dignidade e para contribuir no desenvolvimento da pesquisa dentro dos padrões éticos e científicos.

A pesquisa foi submetida e aprovada pelo Comitê de Ética em Pesquisa (CEP), protocolo no. CAAE: 1.222 .595 .

Os profissionais que aceitaram participar assinaram o Termo de Conscentimento Livre e Escrlarecido (TCLE) e estavam livres para desistir da pesquisa quando desejassem. a qualquer momento. 
Revista Eletrônica Gestão \& Saúde ISSN: 1982-4785

Ferreira PTLA, TeixeiraTCA, Galhardi, NM et al

Resultados

Dos 28 profissionais da equipe de enfermagem que responderam ao questionário, $96 \%$ era do sexo feminino. Quanto à faixa etária, $61 \%$ dos participantes tinha 25 anos ou mais; 14\%, menos de 25 anos; e 18\%, 50 anos ou mais. Com relação ao tempo de trabalho na instituição, treze profissionais (46\%) trabalhavam há menos de cinco anos; nove $(32 \%)$, entre seis e dez anos; e um (4\%), entre 11 e 15 anos, conforme Tabela 1.

Dos profissionais entrevistados, dez $(35,7 \%)$ trabalhavam no período da manhã; doze $(43 \%)$ no da tarde; e seis $(21,3 \%)$ no período da noite.

Tabela 1 - Caracterização dos aspetos profissionais e sociodemográficos dos profissionais de enfermagem participantes, Limeira 2015.

\begin{tabular}{lll}
\hline Variáveis & $\mathrm{n}$ & $(\%)$ \\
\hline Idade & 4 & 14 \\
Menos de 25 anos & 17 & 61 \\
25 anos ou mais & 5 & 18 \\
50 anos ou mais & 2 & 7 \\
Não responderam & & \\
Categoria profissional & 2 & 7 \\
Enfermeiro & 26 & 93 \\
Auxiliar de Enfermagem & & \\
Tempo de serviço & & \\
Até 5 anos & 13 & 46 \\
De 6 a 10 anos & 9 & 32 \\
De 11 a 15 anos & 1 & 4 \\
Não respoderam & 5 & 18 \\
Turno de trabalho & & \\
Manhã & 10 & 36 \\
Tarde & 12 & 43 \\
Noite & 6 & 21 \\
\hline
\end{tabular}

Em relação ao conhecimento dos profissionais sobre o PCS, $16(57 \%)$ conheciam e doze $(43 \%)$ desconheciam o programa. Dentre os profissionais que conheciam, $82 \%$ consideravam o programa como 'extremamente importante'.

Para os respondentes que disseram ter conhecimento prévio do PCS, três $(20 \%)$ afirmaram ter visto por meio da internet; sete $(46 \%)$, por participação em palestras; quatro (26\%), por leitura de artigos; três (20\%), no curso técnico;e um (6\%), no próprio ambiente de trabalho.

Sobre a capacitação por meio de treinamento para o programa, $22(78 \%)$ responedram que receberam e seis $(22 \%)$ disseram não ter recebido treinamento a esse respeito.

Quando questionados se tinham alguma dúvida em relação ao preenchimento da Lista de Verificação ou quanto à 
Revista Eletrônica Gestão \& Saúde ISSN: 1982-4785

Ferreira PTLA, TeixeiraTCA, Galhardi, NM et al

finalidade do programa, 26 (93\%) responderam não ter nenhuma dúvida.

Porém, quand questionadas

especificamente sobre cada item da Lista

de Verificação, sete pessoas listaram

algumas dúvidas, conforme Gráfico 1.

Gráfico 1 - Número de profissionais que apresentaram dúvidas por itens da Lista de Verificação de Segurança Cirúrgica.

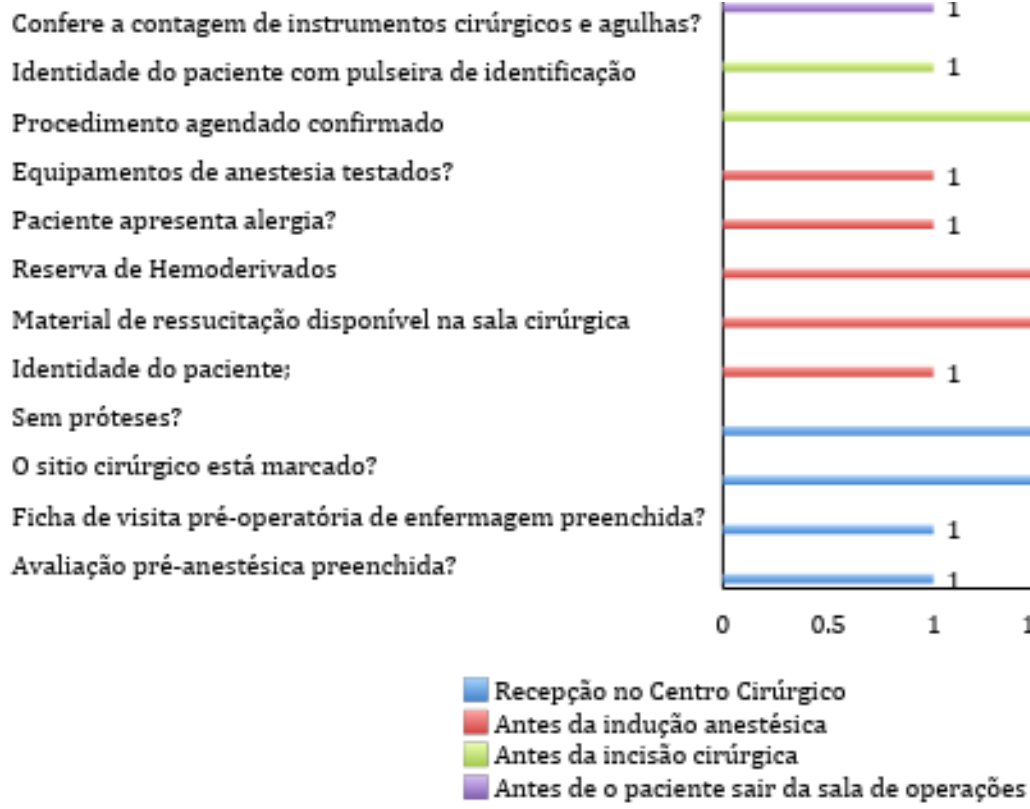

Recepção no Centro Cirúrgico

Antes da indução anestésica

Antes da incisão cirúrgica

Antes de o paciente sair da sala de operações

Ao perguntar se havia alguma discordância em relação aos itens presentes na Lista de Verificação, 16 (58\%) afirmaram que não e onze (39\%), que sim. Os itens dos quais os profissionais discordam constam do Gráfico 2. 
Gráfico 2 - Discordâncias relativas aos itens da Lista De Verificação de Segurança Cirúrgica.

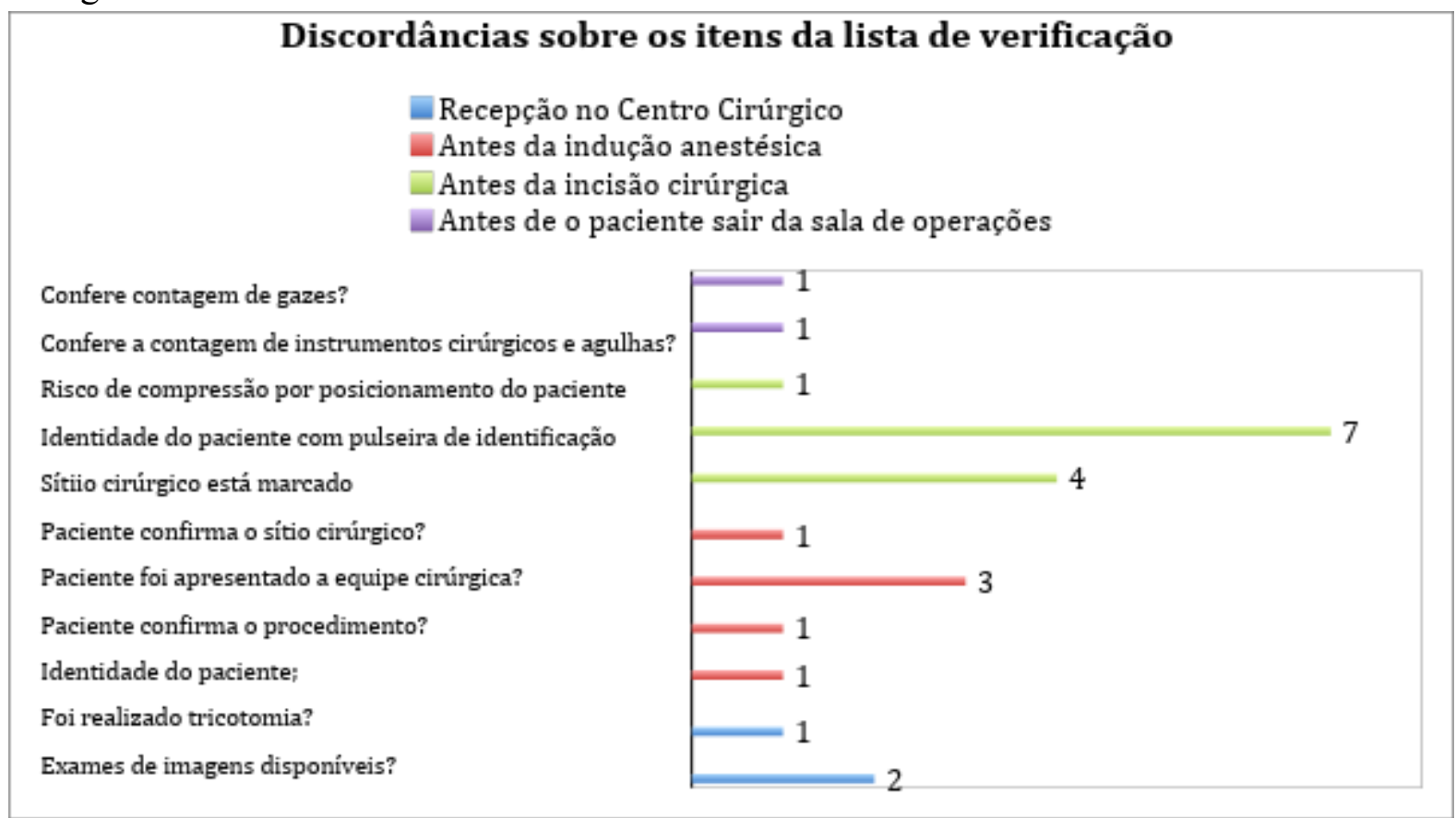

Diante dos itens apresentados no Gráfico 2, os profissionais justificaram a discordândia devido a: ausência de comunicação verbal entre a equipe em relação ao paciente; falta de comunicação com o paciente a fim de checar os dados questionados na Lista de Verificação; ausência de ficha anestésica em cirurgias eletivas; os cirurgiões não se apresentam para os pacientes em sala cirúrgica; falta a colaboração de todos para que a execução do trabalho seja segura; e ausência de tempo para a conferência dos instrumentos antes da cirurgia.

Dos 28 participantes, $22(78 \%)$ consideram o PCS extremamente importante para a prevenção de infecções cirúrgicas; e 23 (79\%) também concordam que o programa é extremamente importante para a prevenção de erros em procedimentos cirúrgicos.

\section{Discussão}

$$
\text { Os resultados mostraram alta }
$$

adesão à pesquisa pela equipe de enfermagem, ou seja, 26 participantes para uma população de 34 .

Em relação ao número de profissionais que conheciam o PCS, notase um percentual mediano, representando $57 \%$ dos participantes. Essa informação mostra que ainda é preciso maior divulgação a respeito desse programa e da importância da Lista de Verificação como uma ferramenta para a prevenção de erros e promoção da segurança dos pacientes submetidos à cirurgia.

Estudo publicado na França em 2011 investigando sobre a implantação da Lista de Verificação observou falta de informação à equipe. Além disso, identificou-se que a Lista de Verificação é mais um documento administrativo suplementar do que algo útil para melhoria da segurança do paciente ${ }^{11}$.

Aquele estudo destaca que, após a implementação do PCS, a porcentagem de profissionais da equipe de enfermagem 
Revista Eletrônica Gestão \& Saúde ISSN: 1982-4785 Ferreira PTLA, TeixeiraTCA, Galhardi, NM et al

que recebeu treinamento para a utilização da Lista de Verificação foi alto.

Dentre as dificuldades para a aplicação da Lista de Verificação, destaca-se a falta de participação da equipe, citada por $32 \%$ dos profissionais, além do tempo dispendido, citado por $17 \%$. Esses resultados vão a favor a outro estudo francês que mostrou como barreira mais comum para a utilização da Lista a falta de participação da equipe, sendo a falta de comunicação a situação mais citada $^{12}$.

A comunicação permeia todas as atividades que integram a assistência ao paciente e quanto mais especializada for o serviço, maior a necessidade de informações técnicas, especializadas e precisas $^{13}$.

É preciso atentar para a integração e interação com a equipe e para a utilização da checagem como meio de comunicação e como um facilitador na assistência ao paciente, visto que o relacionamento interpessoal é o segundo item responsável pelo stress de profissionais de centro cirúrgico, atrás somente de sobrecarga de trabalho ${ }^{14}$.

O tempo é considerado fator importante para a realização de procedimentos seguros aos pacientes. A falta de tempo real para seu desempenho soma-se ao tempo dedicado pelos profissionais em suas atividades laborais, e a sobrecarga dificulta a detecção de complicações. Neste estudo, o tempo despendido para o preenchimento da Lista de Verificação foi a segunda dificuldade referida pelos profissionais. Porém, considera- se que, ao conhecer e compreender a importância do PCS e receberem orientações específicas sobre seu preenchimento, a Lista de Verificação poderá ser considerada uma das etapas do procedimento cirúrgico e, como tal, incluída na rotina organizacional ${ }^{15}$.

No hospital onde foi realizada a coleta de dados, todos os circulantes de sala eram auxiliares de enfermagem. Ainda sobre a realidade do hospital investigado, cabe destacar que o enfermeiro compõe a menor porcentagem da equipe, o que pode influenciar para a sua não participação no processo de checagem, já que está envolvido em outras atividades. O enfermeiro, como gerenciador do cuidado, constitui o principal elo entre a equipe e o paciente, e relatos desses profissionais dão à enfermagem importância fundamental, visto que administra a unidade, lidera a equipe e repassa as informações aos demais profissionais ${ }^{16}$.

A Resolução COFEN no 293/2004 define parâmetros para cálculo do dimensionamento de profissionais de enfermagem e indica para tal a utilização do Escore de Schein/Rensis Likert que, em unidade de centro cirúrgico, deverá ser calculado considerando-se os sítios funcionais, particularidades e realidades citadas acima. Cabe ao enfermeiro, apoiado pelas atribuições que lhe são cabíveis, realizar o levantamento das necessidades do serviço ${ }^{17}$.

É importante destacar os aspectos positivos apresentados pelos participantes quando alegam que o PCS é importante. Em pesquisa realizada com o objetivo de avaliar a implantação de uma Lista de Verificação, onze $(32,4 \%)$ pessoas que compuseram a amostra de 34 participantes afirmaram estar totalmente de acordo com a utilização da Lista de Verificação, treze $(38,2 \%)$ afirmaram concordar um pouco e seis $(17,7 \%)$ discordam do uso. Quanto à importância da aplicação do instrumento para a segurança do paciente, quinze $(44,1 \%)$ afirmaram ser importante ${ }^{18}$. 
Revista Eletrônica Gestão \& Saúde ISSN: 1982-4785 Ferreira PTLA, TeixeiraTCA, Galhardi, NM et al

Com base nos resultados apresentados, que mostram dificuldades de comunicação e necessidade de participação multidisciplinar na aplicação da Lista de Verificação, fazem-se necessárias atualizações contínuas para toda equipe que atua no centro cirúrgico, a fim de garantir a eficácia na aplicação da Lista de Verificação como ferramenta para a segurança do paciente.

Sobre as discordâncias em relação aos itens da Lista de Verificação, cabe destacar que foram mencionadas por mais da metade da amostra (58\%). É importante discutir sobre essas questões, já que a ferramenta é preenchida apenas pelos auxiliares de enfermagem do local. Sugere-se definir o profissional que ficará responsável pela confirmação de cada um dos itens da Lista de Verificação e a quem será delegado o preenchimento.

O cuidado seguro resulta tanto de ações corretas dos profissionais de saúde como de processos e sistemas adequados nas instituições e serviços, e de políticas governamentais regulatórias, exigindo esforço coordenado e permanente ${ }^{16}$.

\section{Conclusão}

Este estudo trouxe como justificativa para sua execução a importância de identificar lacunas a respeito da implantação do PCS em hospitais.

Os resultados permitiram destacar, analisar e refletir sobre as lacunas que ainda permeiam o processo de adesão da Lista de Verificação de Segurança Cirúrgica na rotina do centro cirúrgico. São necessários o planejamento e a implantação de atitudes que contribuam para que a Lista de Verificação seja adotada como importante ferramenta para a segurança do paciente cirúrgico.

Conclui-se que os profissionais demonstram dificuldades de comunicação e necessidade de mudança de comportamento frente às etapas da Lista de Verificação, recomendando-se a retomada de treinamentos com toda a equipe - cirurgiões, anestesiologistas, residentes e profissionais de enfermagem - para exposição dos objetivos do programa e sua importância para a segurança do paciente. Ainda, dar oportunidade a todos os profissionais de expor suas dificuldades, dúvidas, discordâncias e sugestões com vistas a colaborar na readequação dos itens presentes na Lista de Verificação, se necessário.

Este estudo também revela a necessidade do desenvolvimento do compromisso coletivo e a importância do apoio institucional para que a implantação do PCS seja satisfatória para todos os envolvidos.

Recomenda-se que sejam realizados novos estudos a fim de continuar a investigação sobre os resultados da utilização da Lista de Verificação de Segurança Cirúrgica tanto para a melhoria da qualidade da instituição como para a segurança dos pacientes.

Como limitação, tem-se que o instrumento foi aplicado somente à equipe de enfermagem, o que impediu o estudo de contribuir com uma visão da equipe multidisciplinar no ambiente do centro cirúrgico, restringindo os resultados apenas à avaliação da enfermagem.

\section{Referências}

1. Ciampone MHT, Peduzzi M. Planejamento Estratégico como instrumento de gestão e assistência. In: Brasil. Instituto para o Desenvolvimento da Saúde. Universidade de São Paulo. Ministério da Saúde. Manual de Enfermagem: Programa Saúde da Família. São Paulo; 2001. p. 23-28. 
2. World Health Organization. Patient safety. [Internet]. Copenhagen; 2014 [citado 10 mar. 2015]. Disponível em: http://www.euro.who.int/en/healthtopics/Health-systems/patientsafety/patient-safety.

3. Gallotti, RMD. Eventos adversos: o que são?. Rev. Assoc. Med. Bras. 2004; 50(2):114-114.

4. Ursi ES. Galvão CM. Prevenção de lesões de pele no perioperatório: revisão integrativa da literatura. Revista LatinoAmericana de Enfermagem, 2006;14(1):124-31.

5. Organização Mundial da Saúde. Orientações da OMS para a Cirurgia Segura 2009 - Cirurgia Segura Salva Vidas / Organização Mundial da Saúde Direção Geral da Saúde. Versão Portuguesa.2009. 196p.

6. Organização Mundial da Saúde. Segundo desafio global para a segurança do paciente: Manual - cirurgias seguras salvam vidas (orientações para cirurgia segura da OMS) / Organização Mundial da Saúde; Rio de Janeiro: Organização Pan-Americana da Saúde. Ministério da Saúde. Agência Nacional de Vigilância Sanitária, 2009. p.19 e 26.

7. Brasil. Ministério da Saúde. Agência Nacional de Vigilância Sanitária. Assistência Segura: Uma Reflexão Teórica Aplicada à Prática. $1^{\text {a }}$ edição. Brasília (DF): Ministério da Saúde; 2013 [citado 10 mar. 2015]. Disponível em: http://www20.anvisa.gov.br/segurancado paciente/images/documentos/livros/Livro 1-Assistencia_Segura.pdf.

8. Brasil. Ministério da Saúde. Portaria $n^{\circ}$ 529, de $1^{\circ}$ de Abril de 2013. Institui o Programa Nacional de Segurança do Paciente (PNSP). Diário Oficial da República Federativa do Brasil; 2013 [citado 10 mar. 2015]. Disponível em: http://bvsms.saude.gov.br/bvs/saudelegis/ gm/2013/prt0529 01 04 2013.html.

9. Haynes $\mathrm{AB}$, Weiser TG, Berry WR, Lipsitz SR, Breizat AH, Dellinger EP, et al. A surgical safety checklist to reduce morbidity and mortality in a global population. N Engl J Med. 2009;

Rev. Gestão \& Saúde (Brasília) Vol. 10, n. 01, Jan. 2019.
360: 491-9.

10. Maziero ECS. Avaliação da Implantação do Programa Cirurgia Segura em um Hospital de Ensino [dissertação]. Curitiba: Universidade Federal do Paraná; 2012. 104 p.

11. Cunat, C.; Flatin, V.; Viale, J.P. Stratégie de déploiement de la check-list dans un CHU. Ann. fr. anesth. reanim., Paris, v.30, p. 484-488, 2011. Disponível em: http://www.em-

consulte.com/revue/ANNFAR/30/6/table -des-matieres/. Acesso em: 11 jul 2015.

12. Fourcade, A. et al. Barriers to staff adoption of a surgical safety checklist. Qual. saf. health care., Londres, v.21, n.3, p.191-197, 2012. Disponível em: http://qualitysafety.bmj.com/content/earl y/2011/11/07/bmjqs-2011-000094.full. Acesso em: 03 nov 2015.

13. Rede Brasileira de Enfermagem e Segurança do Paciente. Estratégias para a Segurança do Paciente: Manual para Profissionais de Saúde. Porto AlegreEDIPUCRS - 2013, 132p.

14. Pancieri AP, Santos BP, Avila MAG, Braga EM. Checklist de cirurgia segura: análise da segurança e comunicação das equipes de um hospital escola. Rev Gaúcha Enferm. 2013;34(1):71-78.

15. Campos SMCL. Sistemática da assistência da enfermagem perioperatória: percepção de enfermeiros assistenciais. Rev. SOBECC. 2000; 5(.4): 21- 25

16. Fontana RT, Lautert L. A prevenção e o controle de infecções: um estudo de caso com enfermeiras. Rev. Bras. Enferm. 2006; 59 (3): 257-261

17. Brasil. Conselho Regional de Enfermagem de São Paulo. Parecer COREN no 042/2011. Dispõe sobre o Dimensionamento de Pessoal de Enfermagem - aspectos gerais. São Paulo, SP, 07 abr. 2011. GAB no 042, 2011. Disponível em: http://inter.corensp.gov.br/sites/default/fil es/042_2011_dimensionamento_geral.pd f. Acesso em: 10 ago 2015. 
Revista Eletrônica Gestão \& Saúde ISSN: 1982-4785 Ferreira PTLA, TeixeiraTCA, Galhardi, NM et al

18. Gueguen T, Coevoet V, Mougeot M., et al. Déploiement de la check-list «Sécurité du patient au bloc opératoire»

Recebido: 20.01.2017

Revisado: 08.11.2018

Aprovado: 27.11.2018
A Percepção Dos Profissionais De Enfermagem...

dans deux hôspitaux lorrains. Performances et difficultés. Ann. fr. anesth. reanim. Paris, v, 30, p. 489-494, 2011

\section{Participação dos autores}

FERREIRA, PTLA atou na concepção teórica, coleta de dados, análise e interpretação dos dados; Redação do artigo ou revisão crítica relevante do conteúdo intelectual; Aprovação final da versão a ser publicada.

TEIXEIRA, TCA atou na concepção teórica, análise e interpretação dos dados; Redação do artigo ou revisão crítica relevante do conteúdo intelectual; Aprovação final da versão a ser publicada.

GALHARDI, NM atou na concepção teórica, análise e interpretação dos dados; Redação do artigo; Aprovação final da versão a ser publicada.

BARBOSA, AE atou na concepção teórica, análise e interpretação dos dados; Redação do artigo; Aprovação final da versão a ser publicada. 